\title{
Mechanical modeling of palatal snoring
}

\author{
Lixi Huang \\ Peterhouse, University of Cambridge, Cambridge CB2 1RD, England
}

(Received 22 June 1994; revised 22 December 1994; accepted 2 February 1995)

\begin{abstract}
The mechanism of human snoring caused by vibration of the soft palate and the characteristics of the noise are investigated. The soft palate becomes unstable and vibrates violently once the inspiratory flow exceeds a critical speed. The physiological phenomenon is modeled by studying flow over a flexible plate. In determining the stability of this flow, the trailing edge conditions are crucial. It is found that the noise generated in the simple experimental configuration has distinct characteristics found in human snores. For example, there is an antiphase relation between unsteady pressures from the oral and nasal channels and this provides a feature that distinguishes snoring by vibration of the soft palate from that caused by other parts of the human airway.
\end{abstract}

PACS numbers: $43.50 . E d, 43.50 . \mathrm{Nm}, 43.28 . \mathrm{Ra}, 43.70 . \mathrm{Aj}$

\section{INTRODUCTION}

Snoring is generally understood to be the low-frequency and often unpleasant sounds created in the upper parts of the human airway. However, there are many sound qualities, each one arising from different vibrations and no systematic categorization is yet available. We take a very simple representation by first dividing snoring into two categories which might form the basis for further classification. The first category predominantly involves vibration of the soft palate; we call it palatal snoring. The second involves vibration of the pharynx and anatomical sections further down the throat; we call that pharyngeal snoring. The relevant parts of anatomy are shown in Fig. 1. In the present study we restrict ourselves to palatal snoring.

Palatal snoring mainly occurs during inspiration. The mouth is open for many snorers, in which case the soft palate is exposed to airflow on both sides. We built a mechanical model to simulate this type of snoring, leaving cases with the mouth shut to future studies. The essential mechanism of the soft palate vibration is flutter, like that of flags and sails. We studied experimentally the acoustics of the model and compared these with human snores.

\section{MECHANICS}

The flow paths during breathing are indicated in Fig. 1 by broken lines. During inspiration, air is drawn through both mouth and nose into the lung. The oral and nasal airways are separated by the hard palate, lying just above the tongue, then by the flexible soft palate which is attached to the downstream end of the hard palate. This dual passage flow is first modeled by the simplified geometry shown in Fig. 2(a). A rigid channel represents the airway boundary. The rigidity is consistent with the fact that most parts of the upper airway are much more rigid than the soft palate. In our model, a flexible plate represents the soft palate, attached at its leading edge to the splitter plate simulating the hard palate. Flow through the channel is generated by an external sucking mechanism which represents the action of the lung. It is easily demonstrated that there exists a critical flow speed above which the flexible plate loses its stability and under- goes vigorous vibration which produces a loud "snoring" sound. Details of how the flexible plate behaves when stability is lost and what happens just before that point were the focus of our previous numerical and experimental studies. ${ }^{1}$ Here we offer an interpretation of the main features of the instability. We neglect the confining effect of the channel walls because in our experiments their proximity to the palate only had a quantitative influence on the flow. The model for our analysis is therefore that shown in Fig. 2(b): the hard and soft palates are idealized as surfaces of negligible thickness in an otherwise unbounded flow.

The palates resemble to a degree an aerofoil with a control surface at its trailing edge. The aerodynamics of which are well understood. Figure 3 shows a plane flap hinged to an upstream splitter plate, or an aerofoil, which is held still. When the flap is displaced downwards, the flow exerts lifting forces on both plates. ${ }^{2}$ The pressure distribution has a squareroot singularity at the leading edge of the splitter plate and a logarithmic singularity at the hinge position. The pressure

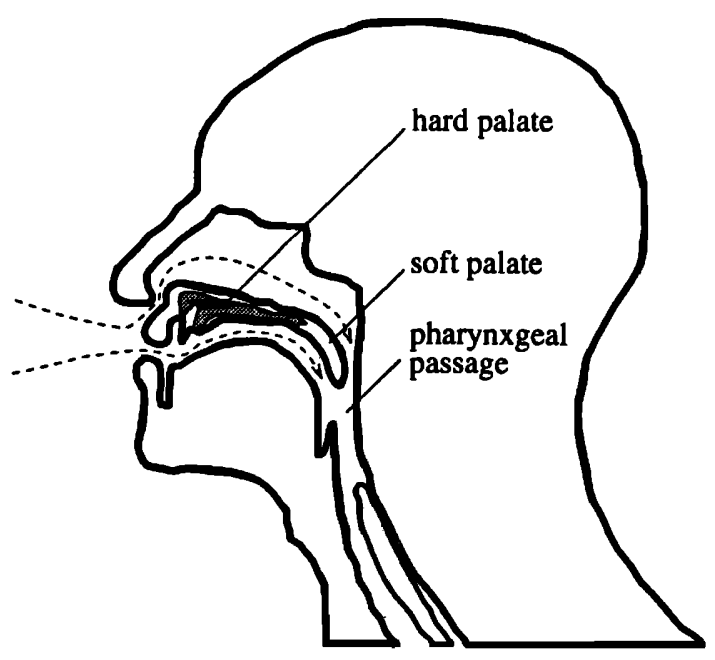

FIG. 1. The anatomy of human upper airway. 
(a) model airway

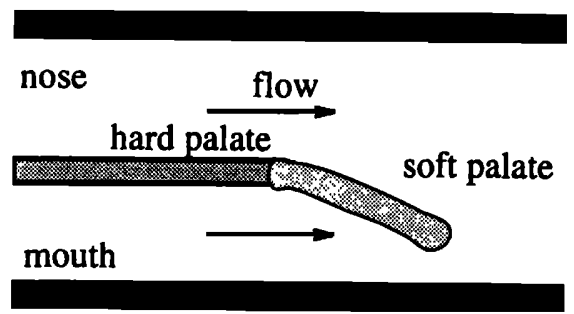

(b) idealized palate model

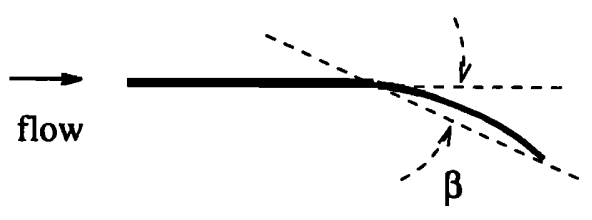

FIG. 2. Two steps of mechanical modeling: (a) model airway and (b) idealized palate model.

on the flap decreases monotonically to vanish at the trailing edge. The total lift on the flap and the rotating moment about the hinge are proportional to the angular displacement of the flap, $\beta$. The pattern of the lift distribution on the cantilevered flexible flap of Fig. 2(b) should be similar apart from the absence of the logarithmic singularity at the leading edge of the flap. In both cases, the lift tends to push the flap back to its undisplaced position parallel with the mean flow. The hinged, rigid flap of Fig. 3 has only one degree of freedom and it is known to be stable, which can be demonstrated easily in the laboratory. A cantilevered, flexible plate is more complicated but it has a vibration mode that involves simple bending at relatively low flow speed. The distribution of the lift is such that it tends to bend the flap with lift force concentrated near the leading edge of the flap. As a result, the upstream part is deflected but the downstream part will follow as soon as bending stresses make themselves felt. We have explained elsewhere that the unsteady part of the lift force plays the role of mechanical damping. ${ }^{1}$ The part of the lift caused by the displacement of the flap is mainly determined by the position of the trailing edge. The motion of the trailing edge lags behind that of upstream parts with the result that the total lift lags behind the overall displacement of the flap. This relative phasing causes energy to flow from the stream into the flap oscillation which grows cycle by cycle. Of course the detailed relationship between lift and displacement also depends on flap curvature but in essence the simple model suffices. The exact form of the lift and the competing structural factors determining the critical flow speed for flutter are beyond the scope of this paper, but those detailed effects do not change the conclusion that the critical flow speed depends mainly on the stiffness of the plate.

To validate the above views and, more importantly, to study the acoustic features of the model, we designed an illustrative experiment based on the model shown in Fig. 2(a). The setup is shown in Fig. 4. A pump was used to suck air through a perspex tube, the flow speed being controlled by a power regulator. Model palates consisted of a hard part made of wood and a flexible part made of sheet rubber 1.6 $\mathrm{mm}$ thick. The rubber plate has a tonguelike shape so that its motion is not obstructed by the tube. There is no reason against using a rectangular palate and doing the experiment in a two-dimensional channel flow. Indeed, in an earlier article $^{1}$ we described such an experiment with the same qualitative results as described below. The rubber was attached to the wood and together inserted vertically into the tube so that gravity played no part in the oscillation. The system lost stability at a definite flow speed and the motion was seen via stroboscopic illustration to be convective and wavelike. Careful observation revealed that the rubber sheet bent before it embarked on violent vibration, eventually hitting both sides of the tube wall. Sound measurements were taken at locations A, B, C, D, I, and Q sketched in Fig. 4(b). At these positions, 1/8-in. B\&K 4135 condenser-type microphones were mounted flush with the inner tube surface. Position $\mathrm{Q}$ was external to the tube, where the noise level was lower and a bigger, more sensitive microphone, $1 / 2$-in. B\&K 4134 , was more appropriate. The rubber palate was found to vibrate above the critical air speed of $15.0 \mathrm{~m} / \mathrm{s}$ and both preand postflutter pressure variations were measured. The analog signals were recorded by a PC through an AT\&T digital signal processor DSP32C together with a DBDADA daughter board with two input channels.

A typical pressure trace measured at $\mathrm{A}$ is given below to show the gradual buildup of noise during the onset of flutter. Figure 5 shows that there were about three to four oscillations before the signal reached its limit cycle featuring sharp impulses. This indicates that the rubber palate underwent flutter instability rather than static divergence. The buildup cycles were featureless low-frequency waves, not nearly as rich in structure as the mature snore.

To demonstrate the dependency of the critical flow speed on the stiffness of the plate, we simply strengthened the rubber palate by sandwiching it with Sellotape. The criti-

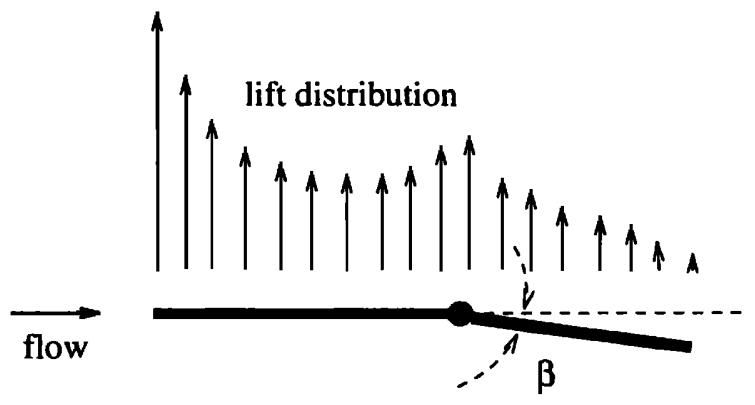

FIG. 3. An aerofoil with a trailing edge control flap. 
(a) front view of the experiment set-up

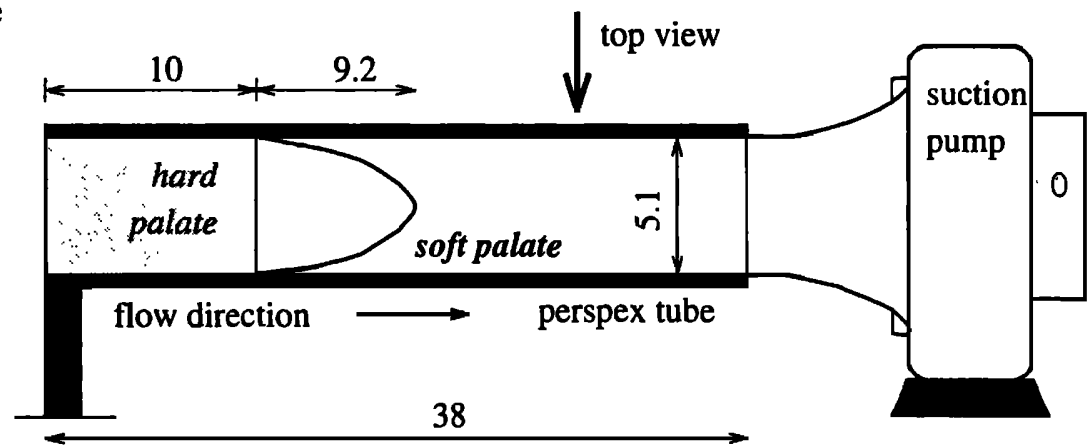

(b) top view of the

microphone positions

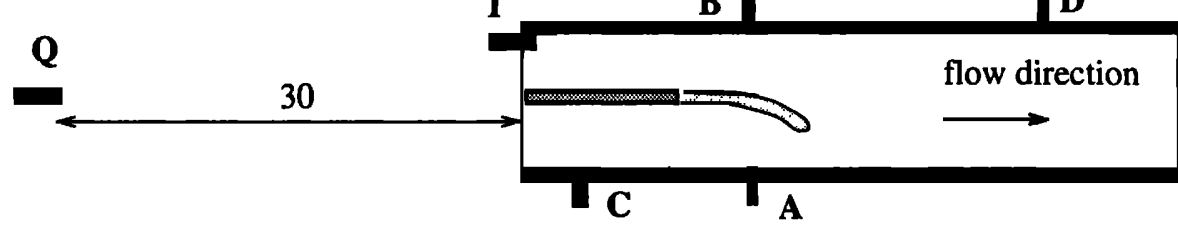

length unit : cm

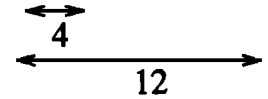

30

FIG. 4. Model experiment setup.

cal speed was found to increase significantly and it easily went above the maximum possible flow generated by the suction pump.

\section{ACOUSTICS}

First, in Fig. 6, we have a "zoom-in" view of the mature fluttering noise. The sharp impulses coincide with the collision of the rubber palate with the tube walls. There are two impulses per cycle, marked $a$ and $b$ in the figure, showing different amplitude levels, the stronger being from collision at the side where the microphone was located.

The pressure variation can be explained by basic hydrodynamics. Consider the time when the rubber palate just crosses the centerline, moving toward the microphone at $\mathrm{A}$, as shown in Fig. 4(b). The passage at A side is narrowing and becomes a nozzle while that at $B$ side is a diffuser. The boundary condition at the trailing edge demands a single pressure there. The "quasisteady" pressure at the upstream

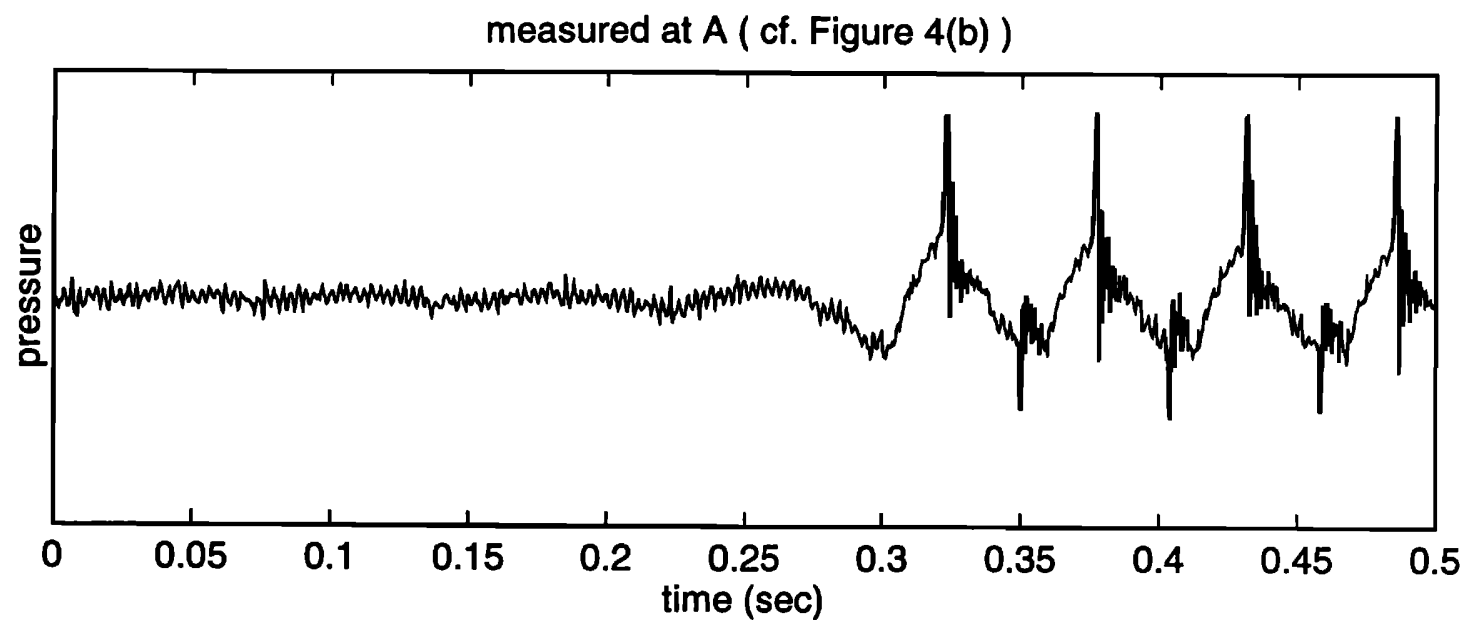

FIG. 5. Pressure signal during flutter onset. 
measured at $A$ (cf. Figure 4(b))

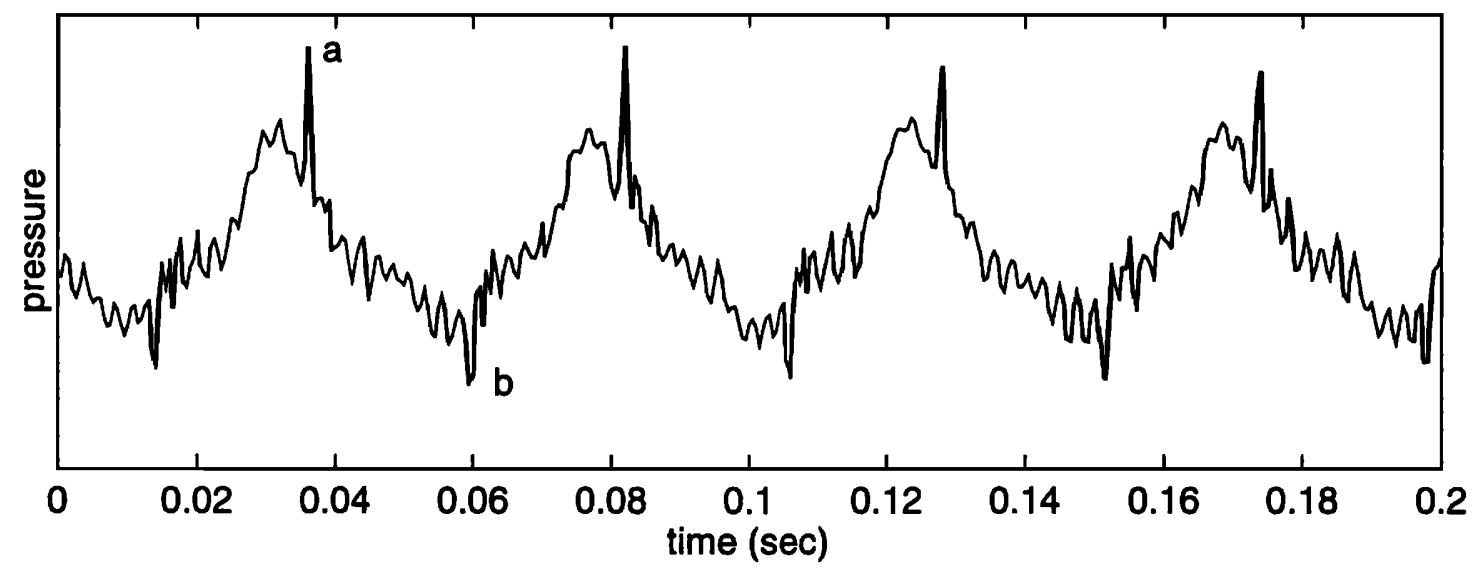

FIG. 6. Zoom-in pressure signal during flutter.

positions is therefore higher at point $\mathrm{A}$ than that at point $\mathrm{B}$. The pressure reaches its peak value close to the time the rubber palate hits the side.

We next consider the impulsive pressure peaks caused by wall impact. When the palate hits the wall of A side, the sign of the pressure felt at point $A$ is positive. That at $B$ at the same moment is the same as the impulse felt at $A$ when the palate hits the other side later; its sign is found to be negative. These two impulses are marked, respectively, $a$ and $b$ in Fig. 6. There are two possible mechanisms for the generation of the pressure impulses. One is by the sudden motion of the membrane following collision and the other is the sudden closure of the air passage at the time of collision.

Sound is generated not by steady motions of objects but by unsteady motions. When a moving plate suddenly stops, the air following the moving surface cannot adjust to the sudden event quickly enough and is compressed while that in the front is expanded. Expansion means lower pressure and vice versa. Pressure impulses of opposite signs propagate away from the two sides of the plate. By applying this reasoning we expect the expansive pulse to give a negative pressure at mark a in Fig. 6 instead of a positive one. Also, we expect a positive impulse at point $B$ with a time delay no longer than that needed for sound to travel the distance of the tube diameter. This is not consistent with the observed time intervals between impulses. Another inconsistent aspect is the 1.75-ms duration of pulse; it is too long. In short, the first mechanism can be excluded.

The second mechanism suggests impulse generation by the sudden closure of the flow passage at collision. As was explained earlier, the pressure is higher in the narrowing passage than in the other. This pressure rise is dramatized when the palate actually hits the wall and partly closes off the passage. It means less volume flow into the passage but this happens only after the pressure rise reaches the passage entrance travelling there as a wave at the speed of sound. Meantime, the axial pressure gradient accelerates the flow through the passage. A negative pressure pulse is created and

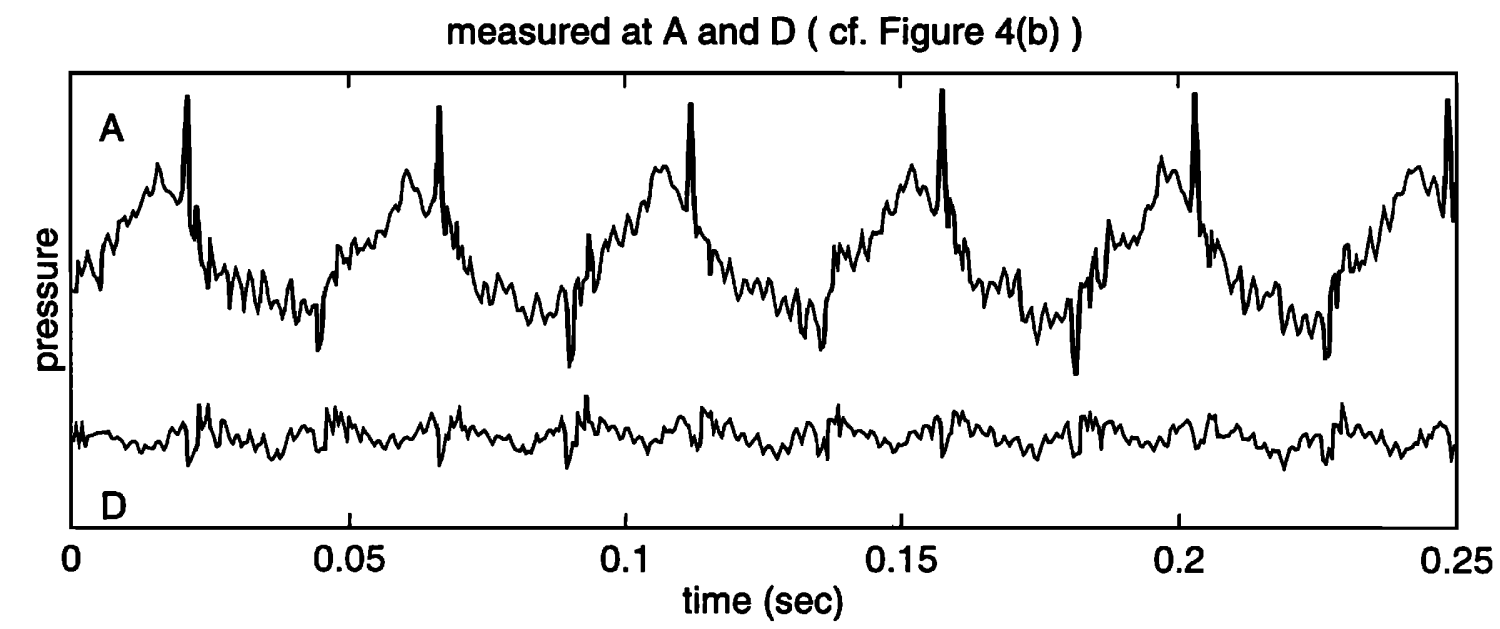

FIG. 7. The relation of sounds heard from up- and downstream. 


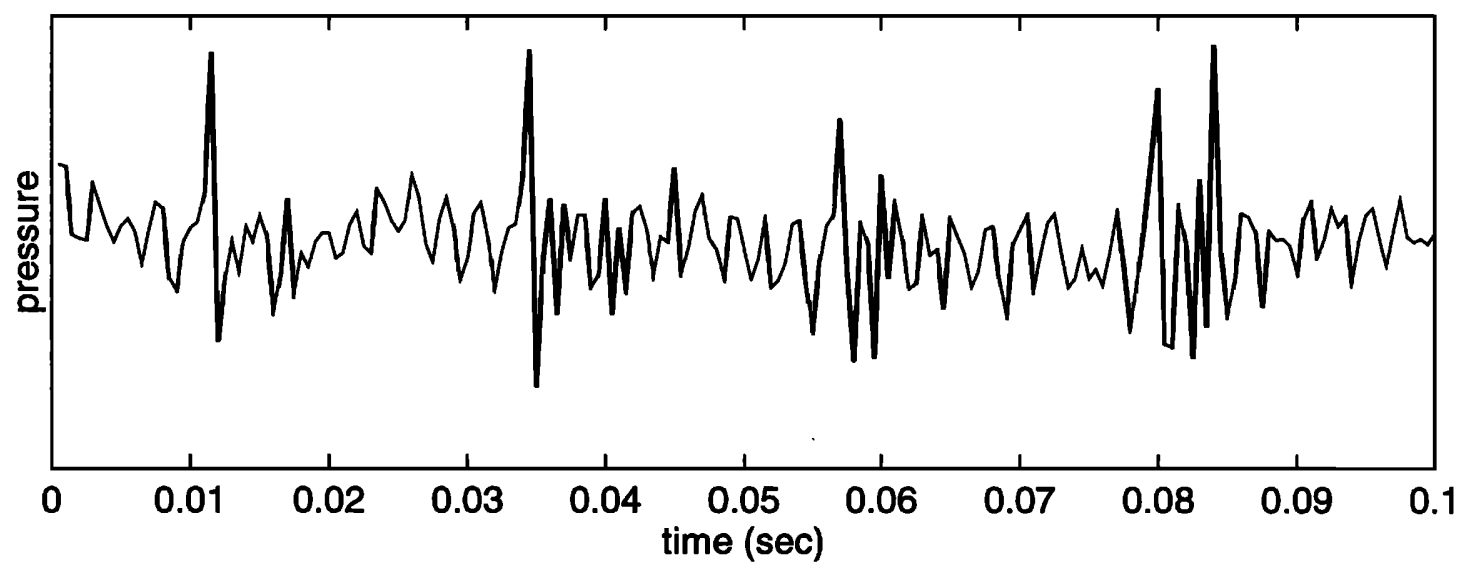

FIG. 8. Sound heard outside the tube.

travels downstream. This acoustic path of impulses is consistent with our experiments. From the analog signal we count the time from when the impulse is felt at point $A$ and find that impulses arrived at $\mathrm{C}, \mathrm{I}$, and $\mathrm{B}$ at retarded times $\tau=0.222,0.500$, and $0.875 \mathrm{~ms}$, respectively. The counting is accurate to the analog sample interval $1 / F_{s}$ where $F_{s}=8$ $\mathrm{kHz}$. The speed of sound is $c=343 \mathrm{~m} / \mathrm{s}$, so the sound travel distance from the collision point to $\mathrm{A}$ and also to the other three points is simply calculated. This gives $7.6 \mathrm{~cm}$ for $\mathrm{A}$ and $\mathrm{C}$, very close to the 8-cm physical distance between the two points. The sound reception at point $I$ is complicated by the geometry but the order of retarded times suggests that the path is followed from $C$ to $I$ and then on to $B$. It gives 12.9-cm sound travel distance while the physical distance is $12 \mathrm{~cm}$. The agreement makes the model quite plausible.

When the palate hits the wall of A side, the pressure impulse felt at position $D$ is negative, bearing the same sign as that at B. But that at $B$ changes to positive when the palate hits the other wall. D remains on the same side of the blocked flow at all times and the pressure pulses at $\mathrm{D}$ are always negative, repeating at twice the frequency of the negative pulses at $\mathrm{A}$ and $\mathrm{B}$. The pressure traces at $\mathrm{A}$ and $\mathrm{D}$ are compared in Fig. 7.

The sound heard outside the tube is of more direct interest to snoring noise simulation. There the sign of the pulses is always positive but their amplitude is much lower. Sound radiation from an open tube depends on the tube radius divided by the wavelength; short waves radiate more effectively. The sound trace at point $Q$ is shown in Fig. 8.

Exact antiphase was found between the pressures at $A$ and B, though the sharp peaks had a small time delay. Figure 9 shows such a pair of signals. This striking though simple feature could well be the most reliable diagnostic aid for distinguishing sound generated by palatal fluttering from that due to pharyngeal wall oscillations. This aspect is now being explored in detail with colleagues in Cambridge.

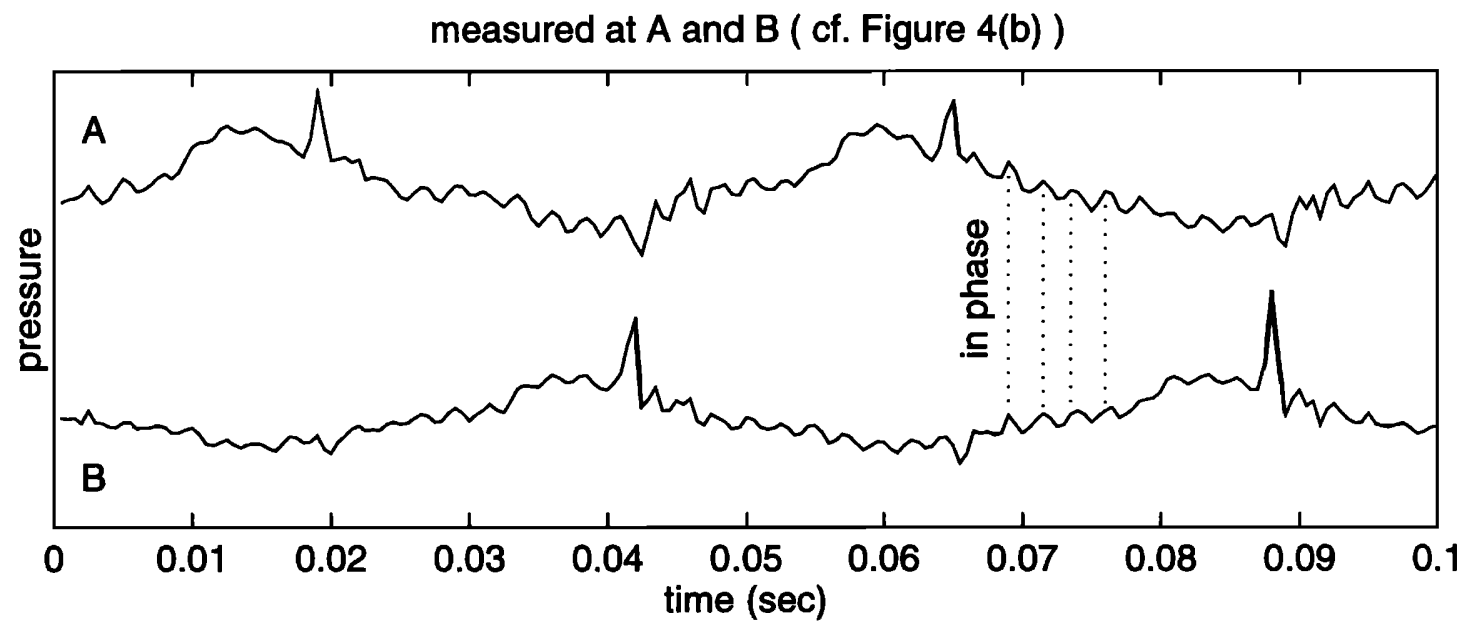

FIG. 9. Antiphase relation of sounds from the dual passages. 


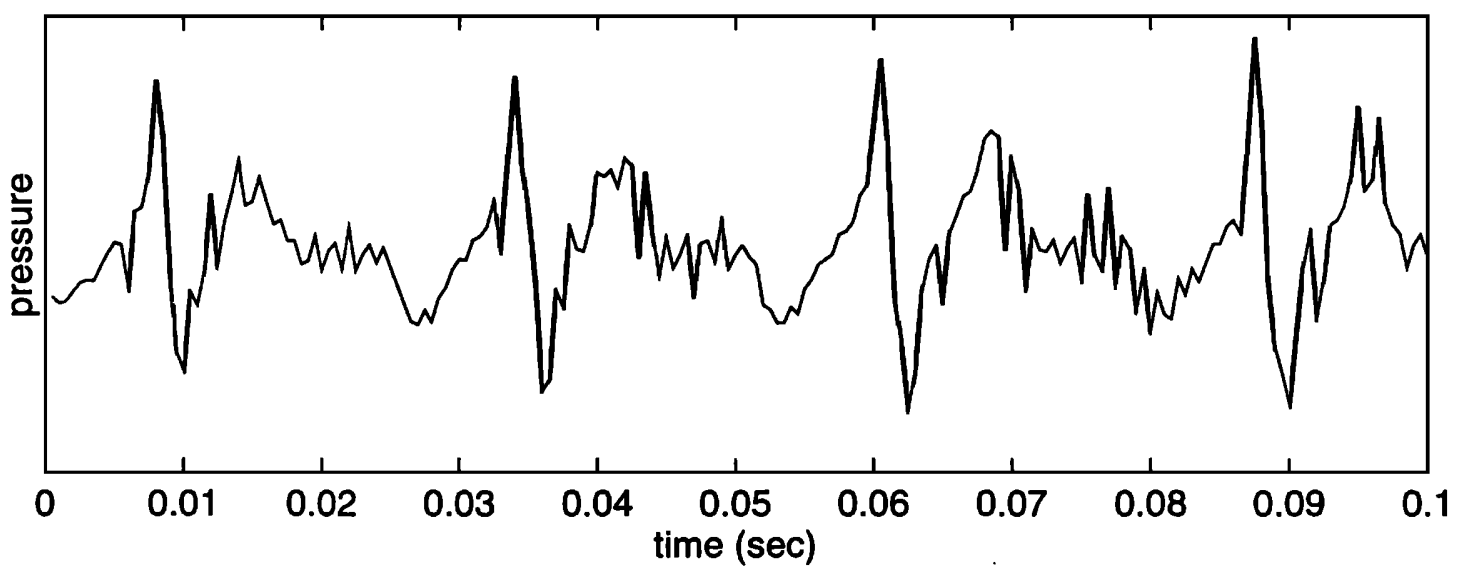

FIG. 10. A typical simulated snore in clinic.

Sound traces from our experiment also show some regular oscillation of higher frequency which are due to the pump. Its amplitude was no bigger than the preflutter level, as can be seen in Fig. 5. This noise is perfectly in phase when measured at points $\mathrm{A}$ and $\mathrm{B}$, as highlighted by the vertical dot lines in Fig. 9.

\section{SNORE SAMPLES}

We now give some human snore samples to relate the mechanical modeling to the real respiratory situation. The snoring was recorded on a digital-analog tape, Sony DAT690 , via the same microphones as used in the model experiments. The recorder had flat frequency response from $2 \mathrm{~Hz}$ to over $20 \mathrm{kHz}$. Figure 10 is a typical simulated snore conducted in the Addenbrooke's snoring clinic with the patient's mouth open; the soft palate was seen vibrating. The noise is found to be a series of pulses repeating every $26.5 \mathrm{~ms}$, i.e., periodic at $37.7 \mathrm{~Hz}$. The waveform is qualitatively similar to the noise heard outside the model experiment, as shown in Fig. 8.

In the second example, we had two simultaneous sound recording channels, one for the mouth and one for the nose. The two microphone sensors were inserted into the oral and nasal entrance up to $5 \mathrm{~mm}$ while the patient undertook a simulated snore. Figure 11 shows two remarkable features. One is the antiphase relation between the two pressures as found in the mechanical modeling; the other is the clear sign of flutter buildup. The impulses are rather less distinctive than those in the model experiment.

Our final example is from another patient's natural sleep state. Figure 12 shows that the time interval between pulses progressively increases from 21.6 to $40.4 \mathrm{~ms}$, which we think is indicative of a relaxing muscle condition during natural snoring. The waveform again looks similar to the model experiment noise.

subject 2

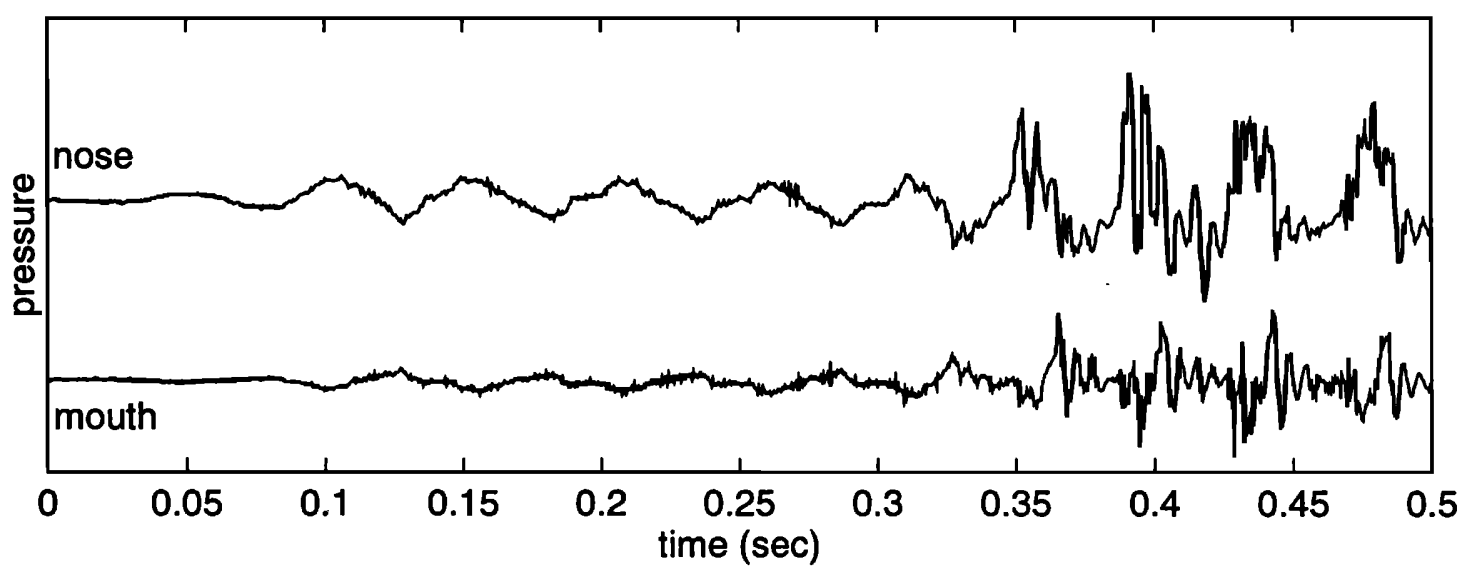

FIG. 11. Antiphase relation during palatal snore buildup. 


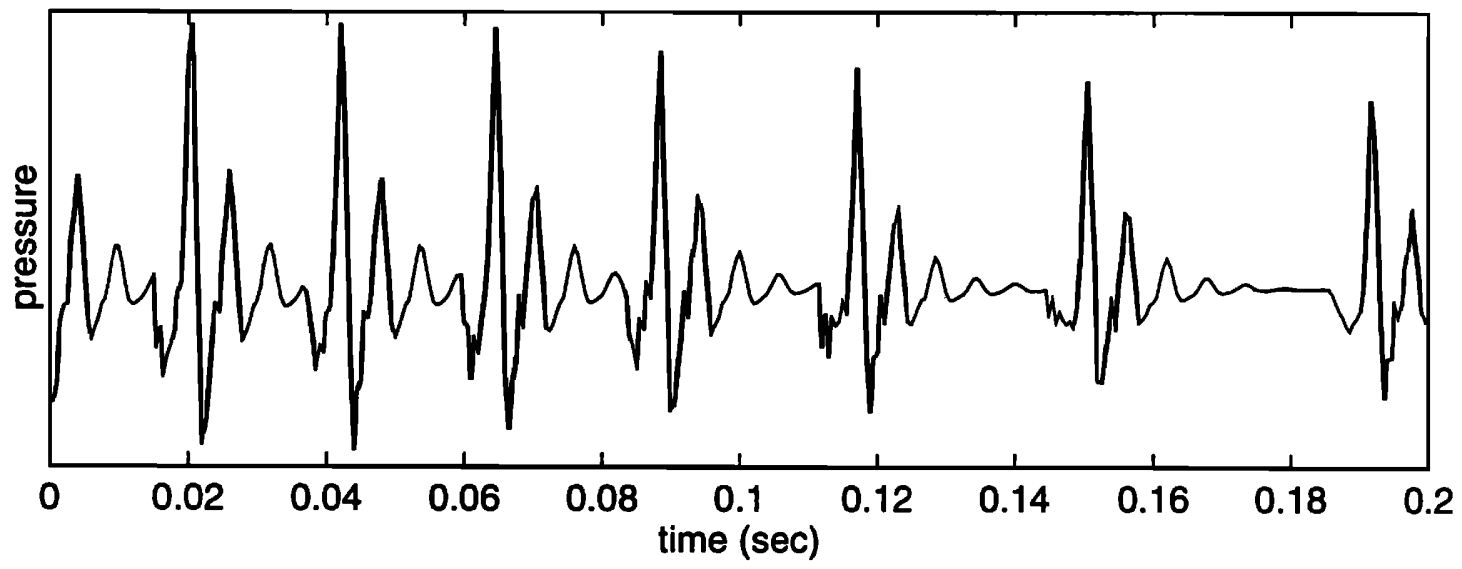

FIG. 12. A snoring sample from natural sleep state.

\section{CONCLUSIONS}

To summarize, we point out that two factors are crucial to the stability of the cantilevered flap that models the soft palate. One is the stiffness of the flap that determines the critical wind speed. Based on our mechanical model, stiffening of soft palate is expected to eliminate palatal snoring. This has been successfully explored in new surgical operations. ${ }^{3}$ The other factor is the phasing of the motion of the trailing edge; a crucial time delay occurs because the distorting forces act upstream and it takes time for the motion to convect to the trailing edge whose attitude sets the aerodynamic condition.

The induced sound has many features which might well be used as a noninvasive diagnosis tool for palatal snoring. In the model experiment, the external sound consists of sharp pulses, repeating at twice the palatal oscillation frequency. The antiphase relation of the pressure variations from the oral and nasal airways is a good test of whether snoring is caused by the soft palate vibration. All these features have been found in real snores known to be palatally induced.

\section{ACKNOWLEDGMENTS}

The author is most grateful for the guidance and help given by Professor J. E. Ffowcs Williams. The author also thanks his two medical colleagues, Peter Ellis and James Quinn, for their cooperation in taking snore samples. Invaluable comments made by referees are also gratefully acknowledged. The work was done while the author received a College Research Fellowship from Peterhouse, Cambridge.

${ }^{1}$ L. Huang, "Flutter of cantilevered plates in axial flow," J. Fluids Struct. 9 (2), 127-147 (1995).

${ }^{2}$ R. L. Bisplinghoff, H. Ashley, and R. L. Halfman, Aeroelastícity (Addison-Wesley, Reading, MA, 1955).

${ }^{3}$ P. D. M. Ellis, J. E. Ffowcs Williams, and J. M. Shneerson, "Surgical relief of snoring due to palatal flutter: A preliminary report," Ann. R. Coll. Surg. Engl. 75, 286-290 (1993). 